\title{
Stability of a Class of Linear Switching Systems with Time Delay
}

\author{
Sehjeong Kim, Sue Ann Campbell, and Xinzhi Liu*†
}

\begin{abstract}
We consider a switching system composed of a finite number of linear delay differential equations (DDE). It has been shown that the stability of a switching system composed of a finite number of linear ordinary differential equations (ODE) may be achieved by using a Common Lyapunov Function Method switching rule. We modify this switching rule for ODE systems to a Common Lyapunov Functional Method switching rule for DDE systems and show that it stabilizes our model. Our result uses a Riccati type Lyapunov functional under a condition on the time delay.
\end{abstract}

Index terms: switching systems, Lyapunov functional, delay differential equations

\section{INTRODUCTION}

A switching system is a type of hybrid system which is a combination of discrete and continuous dynamical systems. The standard model for such systems is given in [1]. These systems arise as models for phenomena which cannot be described by exclusively continuous or exclusively discrete processes. Examples include the control of manufacturing systems $[2,3]$, communication networks, traffic control $[4,5,6]$, chemical processing [7] and automotive engine control and aircraft control [8].

There have been many studies of stability in switching systems (see e.g. [9] and references therein). We note in particular the work of [10] who studied a linear switching system of the form

$$
\dot{x}(t)=M_{i_{k}} x(t), \text { for } t \in\left[t_{k}, t_{k+1}\right), k=0,1,2, \cdots
$$

where $x \in \mathcal{R}^{n}, i_{k} \in\{1,2, \cdots, N\}$ and $M_{i_{k}} \in \mathcal{R}^{n \times n}$. Assuming that there exists a Hurwitz linear convex combination, $F=\sum_{i=1}^{N} \alpha_{i} M_{i}$, they proposed a state dependent switching rule to stabilize (1). Their rule and proof of stability rely on a common Lyapunov function, i.e. a Lyapunov function valid for each subsystem of (1) in some subregion of $\mathcal{R}^{n}$.

\footnotetext{
${ }^{*}$ This research was supported by NSERC.

${ }^{\dagger}$ Sehjeong Kim, Sue Ann Campbell and Xinzhi Liu are with the Department of Applied Mathematics, University of Waterloo, Waterloo, ON N2L 3G1, Canada (email: sacampbell@uwaterloo.ca)
} 
Delay differential equations (DDE's) arise as models for systems where the rate of change of the state depends not only on the current state of the system but also its state at some time(s) in the past (see e.g. $[11,12,13,14])$. This is especially important for control systems where actuators, sensors and transmission lines may introduce time lags [15]. Since many of the applications of switching systems involve control, it is natural to consider the effect of time delays in such systems, i.e. to study switching systems with time delay.

To date, most work in this area has focused on sliding mode control $[16,17,18]$ or on formulating conditions such that the system is stable for any switching rule [19, 20]. The main approach in this latter work is to find a Lyapunov functional which is valid for all subsystems in all of $\mathcal{R}^{n}$. The terminology common Lyapunov functional is also used in this work. Their meaning is different than ours, which follows the definition of [10] for ODE systems, as described above. Other work along similar lines is that of Boukas and Liu [21]. They considered a stochastic model, a Markov jump linear system with time delay that is a switching system whose switching rule is a continuous-time Markov process. They obtained various delay independent and delay dependent stability results using a Lyapunov functional and LMI approach.

By contrast, here we focus on formulating a switching rule that will stabilize a given switching system. One might expect that a switching rule which is designed to stabilize a system with zero delay would still stabilize the system for sufficiently small delay. The goal of this paper is to show this is true and quantify how small the delay must be, for a linear system similar to (1).

The paper is organized as follows. In Section II we formulate our model, propose a switching rule for the system and use a common Lyapunov functional to show it stabilizes the system for sufficiently small delays. In Section III we apply this method to two examples: a system that switches between unstable subsystems and a delayed feedback control system with switching.

\section{SWITCHING SYSTEM WITH TIME DELAY}

\section{A. Model and preliminaries}

Let $\mathcal{C}=C\left([-\tau, 0], \mathcal{R}^{n}\right)$ be a Banach space of continuous functions with the norm

$$
\|\phi\|_{\tau}=\sup _{-\tau \leq \theta \leq 0}\|\phi(\theta)\| .
$$

Given an initial time, $t_{0}$, an initial function, $\phi \in C$, and a switching sequence $\left(i_{0}, t_{0}\right),\left(i_{1}, t_{1}\right), \cdots,\left(i_{j}, t_{j}\right), \cdots$, we consider the following linear switching system with time delay

$$
\begin{cases}\dot{x}=A_{i_{k}} x(t)+B_{i_{k}} x(t-\tau) & \text { for } t \in\left[t_{k}, t_{k+1}\right), k=0,1,2, \cdots \\ x(t)=\phi(t) & \text { for } t_{0}-\tau \leq t \leq t_{0},\end{cases}
$$


where $\tau>0, x \in \mathcal{R}^{n}, A_{i}, B_{i} \in \mathcal{R}^{n \times n}$, and $i_{k} \in\{1,2, \cdots, N\}, 0<N<\infty$. As is usual for delay differential equations, we assume that $\dot{x}$ represents the right-hand derivative of $x$.

Defining $x_{t}(\theta)=x(t+\theta),-\tau \leq \theta \leq 0$, we can write (2) as a nonautonomous functional differential equation

$$
\left\{\begin{array}{l}
\dot{x}=f\left(t, x_{t}\right) \\
x_{t_{0}}=\phi,
\end{array}\right.
$$

where $f:\left[t_{0}, \infty\right) \times \mathcal{C} \rightarrow \mathcal{R}^{n}$ is defined via $f(t, \phi)=A_{i_{k}} \phi(0)+B_{i_{k}} \phi(-\tau)$ for $t \in\left[t_{k}, t_{k+1}\right), k=0,1,2, \cdots$. Note that $f$ is piecewise continuous in $t$ and globally Lipschitz in $\phi$. Using the Method of Steps [22, 23] it is straightforward to show [24] that there is a unique function $x:\left[t_{0}-\tau, \infty\right) \rightarrow \mathcal{R}^{n}$ such that

(i) $x(t)$ satisfies $(3)$,

(ii) $x(t)$ is continuous on $\left[t_{0}-\tau, \infty\right)$,

i.e., that (3) has a unique solution on $\left[t_{0}-\tau, \infty\right)$.

Let $I_{0}=\left[t_{0}, \infty\right)$. We note that $f$ has the following property, which will be used when we prove stability in subsection $C$.

Lemma $1 f$ maps bounded sets of $I_{0} \times \mathcal{C}$ into bounded sets of $\mathcal{R}^{n}$.

Proof Let $D$ be a bounded subset of $C$ and $E=f\left(I_{0}, D\right)$, i.e.,

$$
E=\left\{v \in \mathcal{R}^{n} \mid v=f(t, \phi), \text { for some } \phi \in D, \quad t \in I_{0}\right\}
$$

Since $D$ is bounded, there exists $0<L_{1}<\infty$ such that

$$
\|\phi\|_{\tau} \leq L_{1} \text { for all } \phi \in D
$$

Let $v \in E$, then there exist $\phi \in D$ and $t \in I_{0}$ such that $v=f(t, \phi)$. Let $t \in\left[t_{k}, t_{k+1}\right)$ for some $k \in\{0,1, \ldots\}$. Then

$$
\begin{aligned}
\|v\| & =\|f(t, \phi)\| \\
& \left.=\left\|A_{i_{k}} \phi(0)+B_{i_{k}}\right\| \phi(-\tau)\right) \| \\
& \leq\left\|A_{i_{k}}\right\|\|\phi(0)\|+\left\|B_{i_{k}}\right\| \phi(-\tau) \| . \\
& \leq\left(\left\|A_{i_{k}}\right\|+\left\|B_{i_{k}}\right\|\right)\|\phi\|_{\tau} \\
& \leq\left(\left\|A_{i_{k}}\right\|+\left\|B_{i_{k}}\right\|\right) L_{1} \\
& \leq L
\end{aligned}
$$

where $L=\max _{i=1}^{N}\left(\left\|A_{i}\right\|+\left\|B_{i}\right\|\right) L_{1}$. Thus $E$ is bounded. 


\section{B. Construction of switching regions and a switching rule}

A switching rule is a rule which determines a switching sequence for a given switching system. To design the switching rule for (2) we need the following.

Definition The $i^{\text {th }}$ mode of (2) is the subsystem that is described by the DDE

$$
\dot{x}(t)=A_{i} x(t)+B_{i} x(t-\tau) .
$$

Throughout the rest of the paper, we will assume that there exists a Hurwitz linear convex combination, $F$, of $A_{i}+B_{i}$, i.e.

$$
F=\sum_{i=1}^{N} \alpha_{i}\left(A_{i}+B_{i}\right)
$$

where $0<\alpha_{i}<1$ and $\sum_{i=1}^{N} \alpha_{i}=1$.

Note that when $\tau=0$ system (2) reduces to system (1) with $M_{i}=A_{i}+B_{i}$. We thus use the switching rule defined in [10] for (1) to define the switching rule for (2). The basic idea is as follows: divide $\mathcal{R}^{n}$ into $N$ subregions and use the $i^{\text {th }}$ mode of (2) when $x(t)$ is in the appropriate subregion. Each subregion is defined to make a particular quadratic form negative.

Since $F$ is Hurwitz there exists a positive definite matrix $P$ satisfying

$$
F^{T} P+P F=-Q
$$

for a given positive definite matrix $Q$. Hence, for $x \neq 0$,

$$
x^{T}\left(F^{T} P+P F\right) x=\sum_{i=1}^{N} \alpha_{i} x^{T}\left(\left(A_{i}+B_{i}\right)^{T} P+P\left(A_{i}+B_{i}\right)\right) x=-x^{T} Q x<0 .
$$

Since $0<\alpha_{i}<1$, (9) implies that

$$
\alpha_{i} x^{T}\left(\left(A_{i}+B_{i}\right)^{T} P+P\left(A_{i}+B_{i}\right)\right) x<0
$$

for at least one $i$. So, we construct $\Omega_{i}$ such that

$$
\Omega_{i}=\left\{x \in \mathcal{R}^{n}: x^{T}\left(\left(A_{i}+B_{i}\right)^{T} P+P\left(A_{i}+B_{i}\right)\right) x \leq-x^{T} Q x\right\} .
$$

This leads to the following proposition.

Proposition $2 \mathcal{R}^{n}=\cup_{i=1}^{N} \Omega_{i}$ 
Proof Suppose not, i.e. there exists a $D \subset \mathcal{R}^{n}$ such that $D=\mathcal{R}^{n} \backslash \cup_{i=1}^{N} \Omega_{i}$. Then, for all $x \in D$

$$
x^{T}\left(\left(A_{i}+B_{i}\right)^{T} P+P\left(A_{i}+B_{i}\right)\right) x>-x^{T} Q x \text { for all } i .
$$

Since $0<\alpha_{i}<1$ and $\sum_{i=0}^{N} \alpha_{i}=1$, for $x \in D$

$$
\sum_{i=1}^{N} \alpha_{i} x^{T}\left(\left(A_{i}+B_{i}\right)^{T} P+P\left(A_{i}+B_{i}\right)\right) x>-\sum_{i=1}^{N} \alpha_{i} x^{T} Q x=-x^{T} Q x
$$

But, for all $x \neq 0$

$$
\sum_{i=1}^{N} \alpha_{i} x^{T}\left(\left(A_{i}+B_{i}\right)^{T} P+P\left(A_{i}+B_{i}\right)\right) x=x^{T}\left(F^{T} P+P F\right) x=-x^{T} Q x .
$$

This is a contradiction. Hence, no such D exists and the result follows.

Now, we have $N$ subregions for the given switching system. To prevent a sliding motion (a motion of a trajectory along a boundary between two switching regions) or chattering behaviour (many fast switchings across a boundary) we would like to construct a set of overlapping regions, $\tilde{\Omega}_{i}$, such that each of the $\Omega_{j}$ is contained in exactly one of the $\tilde{\Omega}_{i}$. We thus define

$$
\tilde{\Omega}_{i}=\left\{x \in \mathcal{R}^{n}: x^{T}\left(\left(A_{i}+B_{i}\right)^{T} P+P\left(A_{i}+B_{i}\right)\right) x \leq-\frac{1}{\xi} x^{T} Q x\right\},
$$

for some $\xi>1$, and note that $\Omega_{i} \subset \tilde{\Omega}_{i}$, for each $i \in\{1,2, \cdots, N\}$. We can now describe our switching rule.

Definition (Minimum rule) At each switching we determine the next mode according to the minimum rule:

$$
j(x)=\arg \min x^{T}\left(\left(A_{j}+B_{j}\right)^{T} P+P\left(A_{j}+B_{j}\right)\right) x .
$$

To obtain uniform asymptotic stability of the given switching system we propose the switching rule $\mathrm{S}$ :

S-0 Choose the initial mode, $i_{0}$, by applying the minimum rule to $x\left(t_{0}\right)$.

S-1 Stay in the $i^{t h}$ mode as long as the state $x(t)$ is in $\tilde{\Omega}_{i}$.

S-2 If $x(t)$ hits the boundary of $\tilde{\Omega}_{i}$, use the minimum rule to determine the next mode and switch. 


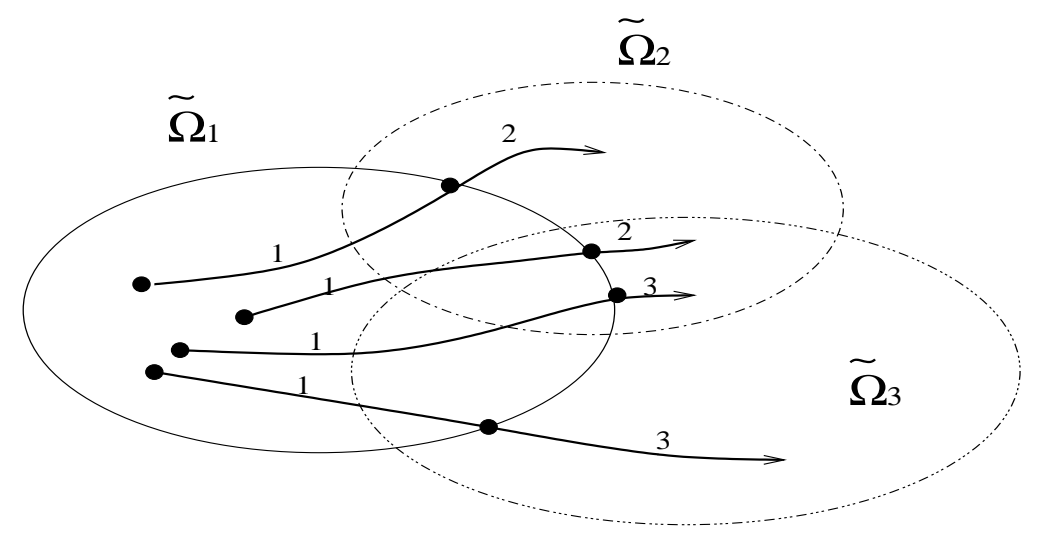

Figure 1: Switching behaviour. Numbers refer to the active mode on the trajectory.

If $\tau=0$, then the work of [10] guarantees the stability of (2) under the switching rule $\mathbf{S}$. In the following we show that stability is preserved for $\tau$ sufficiently small.

The diagram of Figure 1 shows how the switching rule $\mathbf{S}$ works. We start with various initial points in the mode 1 , i.e. $\tilde{\Omega}_{1}$. Then, when the boundary of the mode 1 is reached, i.e. the boundary of $\tilde{\Omega}_{1}$ which intersects with the mode $2\left(\tilde{\Omega}_{2}\right)$ and the mode $3\left(\tilde{\Omega}_{3}\right)$, the system switches to the mode 2 or mode 3 according to the minimum rule S- 0 .

\section{Stability: Common Lyapunov Functional Method}

We define Lyapunov functional $V\left(x_{t}\right)$ as

$$
V\left(x_{t}\right)=x^{T}(t) P x(t)+\int_{\tau}^{2 \tau} d s \int_{t-s}^{t} x^{T}(u) R x(u) d u+\int_{0}^{\tau} d s \int_{t-s}^{t} x^{T}(v) S x(v) d v
$$

where $P$ is as in (12), $R$ and $S$ are some positive definite matrices and $\tau>0$. Now $V$ satisfies

$$
\alpha(\|x(t)\|) \leq V\left(x_{t}\right) \leq \beta\left(\left\|x_{t}\right\|_{\tau}\right)
$$

with

$\alpha(\|x(t)\|)=\lambda_{\min }(P)\|x(t)\|^{2}, \quad$ and $\quad \beta\left(\left\|x_{t}\right\|_{r}\right)=\left(\lambda_{\max }(P)+\frac{3 \tau^{2}}{2} \lambda_{\max }(R)+\frac{\tau^{2}}{2} \lambda_{\max }(S)\right)\left\|x_{t}\right\|_{r}^{2}$.

Note that $\alpha(\cdot)$ and $\beta(\cdot)$ are continuous and increasing functions with $\alpha(0)=\beta(0)=0$. Following the usual procedure for delay differential equations $[22,23]$, we define the right-hand derivative of $V$ along a solution by

$$
\dot{V}\left(x_{t}\right)=\lim _{h \mapsto 0^{+}} \sup \frac{1}{h}\left[V\left(x_{t+h}\right)-V\left(x_{t}\right)\right]
$$

where $x(t)$ is a solution of (2). 
We will show stability by showing that the switching rule defined above guarantees $\dot{V}<0$ along any non-zero solution.

To begin, we follow Kolmanovskii [23] and transform the model. This will allow us to derive a condition on the delay which guarantees $\dot{V}<0$ in each region $\tilde{\Omega}_{i} \backslash\{0\}$, $i=1,2, \cdots, N$.

Assume that the system is in the $i^{t h}$ mode for $t \in\left[t_{k}, t_{k+1}\right)$. Adding and subtracting $B_{i} x(t)$ to (6) we have

$$
\begin{aligned}
\dot{x}(t) & =A_{i} x(t)+B_{i} x(t-\tau)+B_{i} x(t)-B_{i} x(t) \\
& =\left(A_{i}+B_{i}\right) x(t)+B_{i}(x(t-\tau)-x(t)) \\
& =\left(A_{i}+B_{i}\right) x(t)-B_{i} \int_{t-\tau}^{t} \dot{x}(s) d s
\end{aligned}
$$

Assume that the system switched to the $i^{t h}$ mode from the $j^{\text {th }}$ mode at $t=t_{k}$ and that $(t-\tau) \in\left[t_{k-1}, t_{k}\right]$. Then, for $t \in\left[t_{k}, t_{k}+\tau\right)$

$$
\begin{aligned}
\dot{x}(t)=\left(A_{i}+B_{i}\right) x(t) & -B_{i} \int_{t-\tau}^{t_{k}}\left(A_{j} x(s)+B_{j} x(s-\tau)\right) d s \\
& -B_{i} \int_{t_{k}}^{t}\left(A_{i} x(s)+B_{i} x(s-\tau)\right) d s,
\end{aligned}
$$

while for $t \in\left[t_{k}+\tau, t_{k+1}\right)$

$$
\dot{x}(t)=\left(A_{i}+B_{i}\right) x(t)-B_{i} \int_{t-\tau}^{t}\left(A_{i} x(s)+B_{i} x(s-\tau)\right) d s .
$$

Thus, due to the time delay, for the first part of the time interval when the system is in the $i^{t h}$ mode, the evolution of $x(t)$ depends on both the $i^{t h}$ and $j^{t h}$ subsystems. We refer to this as a mixed mode and illustrate it in Figure 2. Note that if $t_{k}+\tau>t_{k+1}$ then the mixed mode will cover the entire interval $\left[t_{k}, t_{k+1}\right)$, i.e. (16) will apply for $t \in\left[t_{k}, t_{k+1}\right)$.

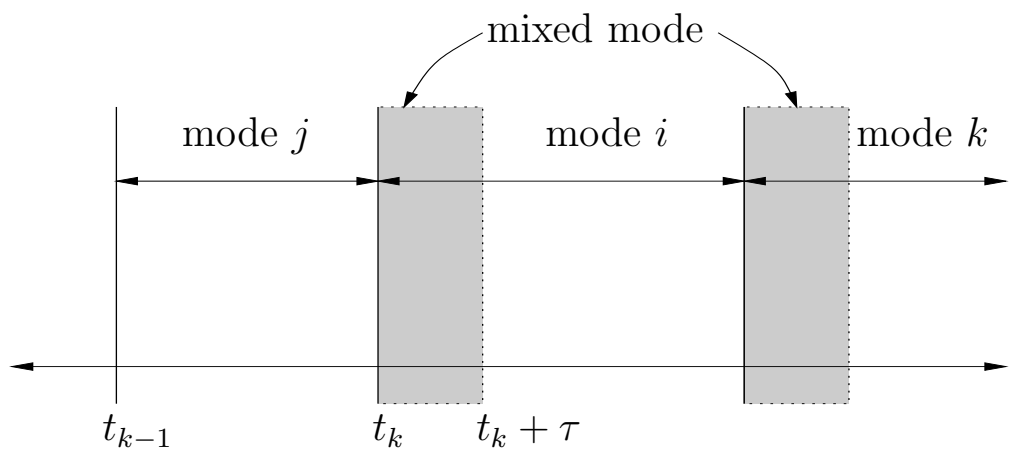

Figure 2: Mixed mode

More generally, if $(t-\tau) \in\left[t_{k-m}, t_{k-m+1}\right]$ and mode $p_{j}$ is active on $\left[t_{k-j}, t_{k-j+1}\right)$, $j=1,2, \ldots m$, then for $t \in\left[t_{k}, t_{k}+\tau\right)\left(\right.$ or $t \in\left[t_{k}, t_{k+1}\right)$, if $\left.t_{k}+\tau>t_{k+1}\right)$

$$
\begin{aligned}
\dot{x}(t)=\left(A_{i}\right. & \left.+B_{i}\right) x(t)-B_{i} \int_{t-\tau}^{t_{k-m+1}}\left[A_{p_{m}} x(s)+B_{p_{m}} x(s-\tau)\right] d s \\
& \quad-B_{i} \sum_{j=1}^{m-1} \int_{t_{k-j}}^{t_{k-j+1}}\left[A_{p_{j}} x(s)+B_{p_{j}} x(s-\tau)\right] d s-B_{i} \int_{t_{k}}^{t}\left[A_{i} x(s)+B_{i} x(s-\tau)\right] d s
\end{aligned}
$$

while for $t \in\left[t_{k}+\tau, t_{k+1}\right) \dot{x}(t)$ is given by (17). This leads us to following proposition. 
Proposition 3 Let

$$
\begin{aligned}
\tilde{\Omega}_{i} & =\left\{x \in \mathcal{R}^{n}: x^{T}\left(\left(A_{i}+B_{i}\right)^{T} P+P\left(A_{i}+B_{i}\right)\right) x \leq-\frac{1}{\xi} x^{T} Q x\right\} \\
\lambda_{\text {max }}^{i} & =\max _{j=1}^{N} \lambda_{\max }\left(P B_{i}\left(A_{j} S^{-1} A_{j}^{T}+B_{j} R^{-1} B_{j}^{T}\right) B_{i}^{T} P\right), \\
L & =\max _{i=1}^{N}\left(\lambda_{\max }(R)+\lambda_{\max }(S)+\lambda_{\text {max }}^{i}\right),
\end{aligned}
$$

and $P$ satisfy $F^{T} P+P F=-Q$, for some $Q>0$.

Suppose that the $i^{\text {th }}$ mode is active and $x(t) \in \tilde{\Omega}_{i}$ on $\left[t_{k}, t_{k+1}\right)$. If

$$
\tau<\frac{\lambda_{\min }(Q)}{\xi L}
$$

then there exists a continuous, increasing function, $\gamma: \mathcal{R}^{+} \rightarrow \mathcal{R}^{+}$, satisfying $\gamma(0)=0$ and $\gamma(s)>0$ for $s>0$ such that

$$
\dot{V}\left(x_{t}\right) \leq-\gamma\left(\left\|x_{t}(0)\right\|\right), \text { for } t \in\left[t_{k}, t_{k+1}\right) \text {. }
$$

Proof The proof can be found in Appendix A.

Proposition 3 ensures that $\dot{V} \leq-\gamma\left(\left\|x_{t}(0)\right\|\right)$ if the $i^{\text {th }}$ mode is used when $x \in \tilde{\Omega}_{i}$. However, it does not guarantee stability of the whole switching system. To do this, we need an appropriate switching rule.

Theorem 4 Let the delay $\tau$ satisfy the condition in Proposition 3. Then, the switching system (2) with switching rule $\boldsymbol{S}$ is uniformly asymptotically stable.

Proof Recall that (9) implies that at each $x \in \mathcal{R}^{n}, x^{T}\left(\left(A_{i}+B_{i}\right)^{T} P+P\left(A_{i}+B_{i}\right)\right) x<0$ for at least one $i$. Thus if the minimum rule chooses mode $j$ then $x(t) \in \tilde{\Omega}_{j}$. Let the initial time $t_{0}$ and initial function $\phi$ be given. Then S-0 determines the initial mode $i_{0}$, so that $x\left(t_{0}\right) \in \tilde{\Omega}_{i_{0}}$. Applying S-1 and Proposition 3 then shows that $\dot{V}\left(x_{t}\right) \leq-\gamma\left(\left\|x_{t}(0)\right\|\right)$ for $t \in\left[t_{0}, t_{1}\right)$. Now consider any interval between switching times, $\left[t_{k}, t_{k+1}\right)$ for some $k>0$. Applying S-2 at $t_{k}$ chooses mode $i_{k}$ such that $x\left(t_{k}\right) \in \tilde{\Omega}_{i_{k}}$. Applying S-1 and Proposition 3 then show that $\dot{V}\left(x_{t}\right) \leq-\gamma\left(\left\|x_{t}(0)\right\|\right)$ for $t \in\left[t_{k}, t_{k+1}\right)$. Thus $\dot{V}\left(x_{t}\right) \leq-\gamma\left(\left\|x_{t}(0)\right\|\right)$ for $t \geq t_{0}$. Recalling the properties of $V(14), \gamma$ (Proposition 3) and $f$ (Lemma 1), the rest of the proof is similar to the proof of Theorem 2.1 in [22, Chapter 5]. 
Remark The multiple delay case, i.e. the case when the $i^{\text {th }}$ mode of the system is given by

$$
\dot{x}(t)=A_{i} x(t)+B_{i} x\left(t-\tau_{i}\right),
$$

can be handled with a similar approach to that described in this section. In this case, the Lyapunov functional will contain a sum of terms of the form

$$
\int_{\tau_{i}}^{\tau_{i}+\tau} d s \int_{t-s}^{t} x^{T}(u) R x(u) d u+\int_{0}^{\tau_{i}} d s \int_{t-s}^{t} x^{T}(v) S x(v) d v
$$

where $\tau=\max _{i} \tau_{i}$.

\section{EXAMPLES}

\section{Example 1: Switching between unstable subsystems.}

Consider the switching system given by

$$
\dot{x}(t)=A_{i} x(t)+B_{i} x(t-\tau), i=1,2
$$

where

$$
\begin{aligned}
& \left(A_{1}, B_{1}\right)=\left(\left[\begin{array}{cc}
-2 & 2 \\
-20 & -2
\end{array}\right],\left[\begin{array}{cc}
-1 & -7 \\
23 & 6
\end{array}\right]\right) \\
& \left(A_{2}, B_{2}\right)=\left(\left[\begin{array}{cc}
-2 & 10 \\
-4 & -2
\end{array}\right],\left[\begin{array}{cc}
4 & -5 \\
1 & -8
\end{array}\right]\right)
\end{aligned}
$$

It is easy to check that each $A_{i}+B_{i}$ is unstable. In particular, $A_{1}+B_{1}$ has a pair of complex conjugate eigenvalues with positive real part and $A_{2}+B_{2}$ has one positive and one negative eigenvalue. This behaviour is illustrated in Figure 3.

There is a Hurwitz convex combination given by

$$
F=\frac{3}{5}\left(A_{1}+B_{1}\right)+\frac{2}{5}\left(A_{2}+B_{2}\right)=\left[\begin{array}{cc}
-1 & 1 \\
-3 / 5 & -8 / 5
\end{array}\right] .
$$

Hence, for $Q=\frac{1}{3} I$ there exists a positive definite matrix $P$ such that $F^{T} P+P F=-Q$, viz.

$$
P=\left[\begin{array}{cc}
64 / 429 & -25 / 858 \\
-25 / 858 & 35 / 286
\end{array}\right]
$$

Following the procedure in section II, we set up $\tilde{\Omega}_{i}$ with $\xi=1.5$ and $Q=\frac{1}{3} I$ as

$$
\tilde{\Omega}_{i}=\left\{x \in \mathcal{R}^{2} \mid x^{T}\left(\left(A_{i}+B_{i}\right)^{T} P+P\left(A_{i}+B_{i}\right)\right) x \leq-\frac{1}{\xi} x^{T} Q x\right\} .
$$




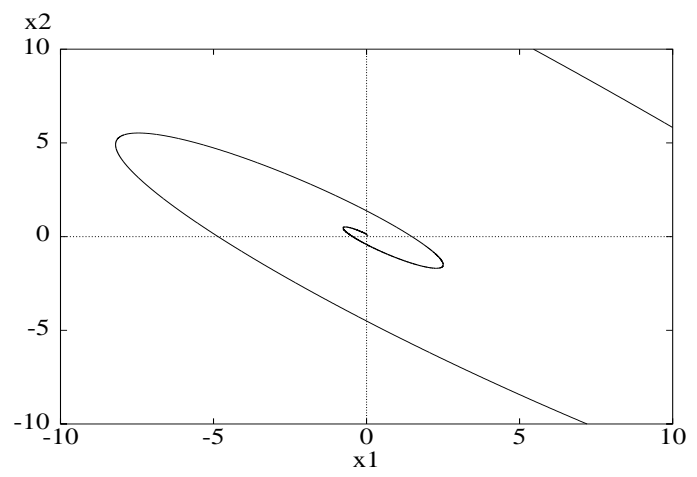

(a)

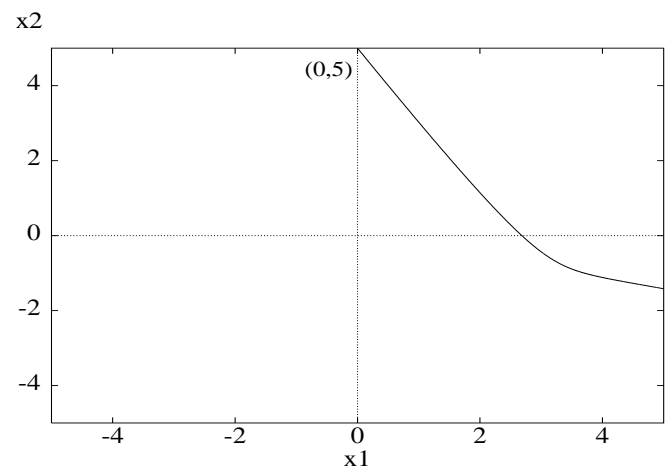

(b)

Figure 3: Behaviour of modes of system (19) when $\tau=0$. (a) Mode 1 with initial value $(0.05,0.05)$. (b) Mode 2 with initial value $(0,5)$.

This gives

$$
\begin{aligned}
& \tilde{\Omega}_{1}=\left\{x \in \mathcal{R}^{2} \mid(545 / 429) x_{2}^{2}-(350 / 429) x_{1} x_{2}-(153 / 143) x_{1}^{2} \leq-\frac{1}{4.5}\left(x_{1}^{2}+x_{2}^{2}\right)\right\}, \\
& \tilde{\Omega}_{2}=\left\{x \in \mathcal{R}^{2} \mid-(1175 / 429) x_{2}^{2}+(175 / 143) x_{1} x_{2}+(331 / 429) x_{1}^{2} \leq-\frac{1}{4.5}\left(x_{1}^{2}+x_{2}^{2}\right)\right\} .
\end{aligned}
$$

These switching regions are illustrated in Figure 4.

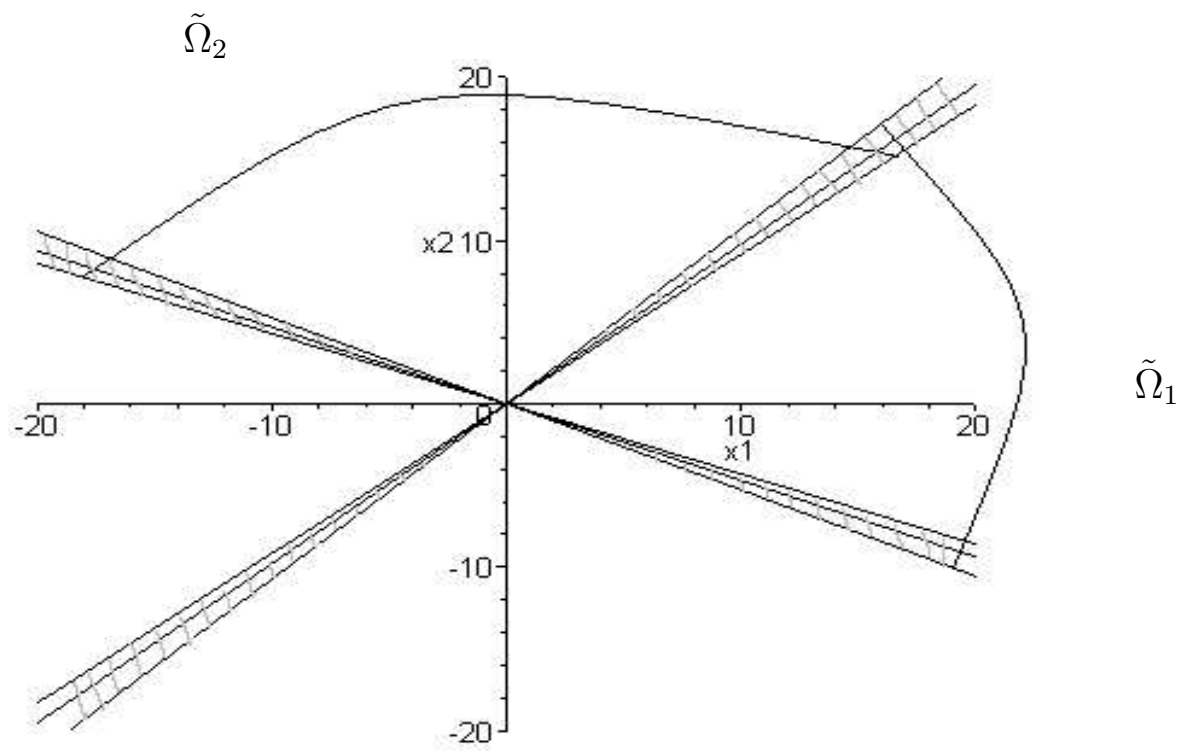

Figure 4: Switching regions for (19): the shaded areas are overlapping regions.

By choosing $R=S=28 I$ with $\xi=1.5$ we find that the critical delay of Proposition 3 is $\tau_{c} \approx 0.001573$. Then, according to Theorem 4 , system (19) with the switching rule $\mathbf{S}$ is guaranteed to be asymptotically stable for any $\tau<\tau_{c}$. This is confirmed by numerical 
simulations, an example of which is shown in Figure 5(a). Note that stability is achieved by entering and remaining in one of the overlapping regions.

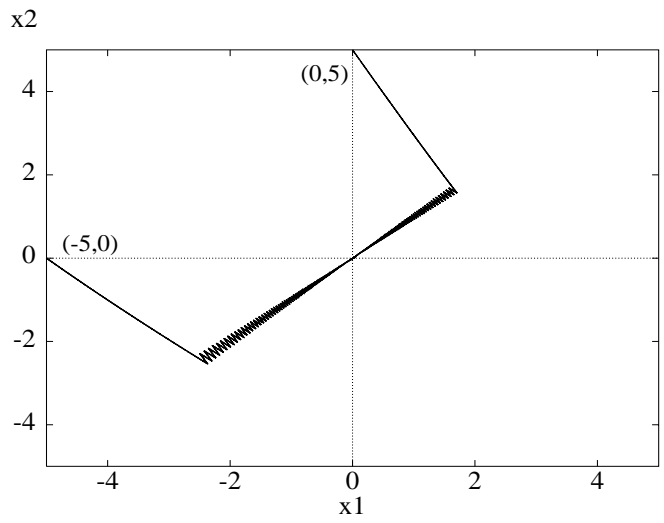

(a)

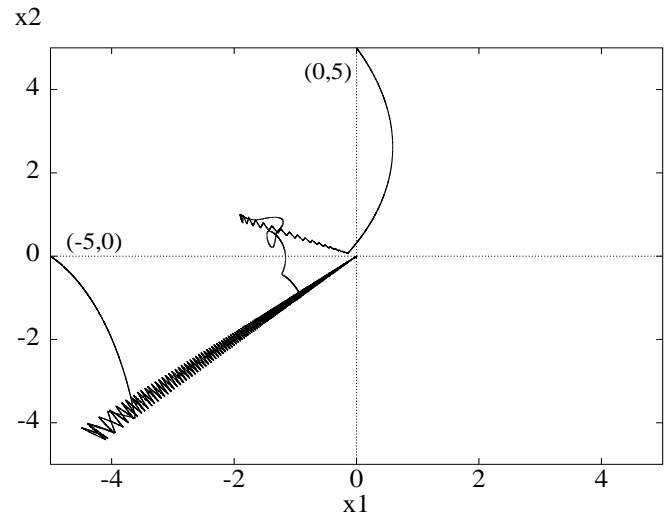

(b)

Figure 5: System (19) with proposed switching rule and various time delays: (a) $\tau=$ $0.001572<\tau_{c}$, (b) $\tau=0.15>\tau_{c}$. Initial condition: $\left(x_{1}(t), x_{2}(t)\right)=\left(x_{10}, x_{20}\right),-\tau \leq t \leq 0$ with two choices of $x_{10}, x_{20}$ as indicated on plot.

Numerical simulations indicate that stability persists for $\tau>\tau_{c}$, however, as can be seen in Figure 5(b), for $\tau>0.1$ the system exhibits irregular transient behaviour. As $\tau$ increases these transients become large enough that the stability would effectively be lost in a physical system. The transient behaviour can be explained by the eigenvalue structure of the delayed subsystems.

\section{Example 2. A switching system with delayed feedback control.}

Feedback control is one of the powerful tools of engineering and a time delay in the feedback is commonly observed. Here, we consider a switching system with delayed feedback control defined as

$$
\dot{x}(t)=A_{i} x(t)+B_{i} u(t-\tau), \text { for } t \in\left[t_{k}, t_{k+1}\right), k=0,1,2, \cdots,
$$

where $x \in \mathcal{R}^{n}, i \in \mathcal{Q}=\{1, \cdots, N\} A_{i} \in \mathcal{R}^{n \times n}, B_{i} \in \mathcal{R}^{n \times p}, u \in \mathcal{R}^{p}$, and $\tau>0$ a constant. We assume that, for each $i \in \mathcal{Q}$,

i) $A_{i}$ is an unstable matrix

ii) $\operatorname{rank}\left(R\left(A_{i}, B_{i}\right)\right)=n$, i.e. the system (22) is controllable. Hence, there exits $K_{i} \in \mathcal{R}^{p \times n}$ such that $A_{i}+B_{i} K_{i}$ is Hurwitz for the system (2) with $\tau=0$. Then, we choose a control by

$$
u(t)=K_{i} x(t)
$$


Therefore we rewrite the original system as

$$
\dot{x}(t)=A_{i} x(t)+B_{i} K_{i} x(t-\tau), \text { for } t \in\left[t_{k}, t_{k+1}\right), \quad k=0,1,2, \cdots
$$

Note that the first part of assumption $i i$ ) ensures that the existence of a Hurwitz linear convex combination of $A_{i}+B_{i} K_{i}$,

$$
F=\sum_{i=1}^{N} \alpha_{i}\left(A_{i}+B_{i} K_{i}\right)
$$

It follows from section II that the switching rule $\mathbf{S}$ with

$$
\begin{aligned}
& \text { Mode } i: \dot{x}(t)=A_{i} x(t)+B_{i} K_{i} x(t-\tau) \\
& \tilde{\Omega}_{i}=\left\{x \in \mathcal{R}^{n}: x^{T}\left(\left(A_{i}+B_{i} K_{i}\right)^{T} P+P\left(A_{i}+B_{i} K_{i}\right)\right) x \leq-\frac{1}{\xi} x^{T} Q x\right\}
\end{aligned}
$$

will achieve uniform asymptotic stability of (23).

The following numerical example illustrates the application described above. Consider (22) with $N=2$ and controllable pairs

$$
\begin{aligned}
& \left(A_{1}, B_{1}\right)=\left(\left[\begin{array}{cc}
3 & 2 \\
-5 & -1
\end{array}\right],\left[\begin{array}{cc}
-1 & 0 \\
1 & -1
\end{array}\right]\right) \\
& \left(A_{2}, B_{2}\right)=\left(\left[\begin{array}{cc}
-1 & 20 \\
-2 & 2
\end{array}\right],\left[\begin{array}{cc}
1 & 1 \\
0 & -1
\end{array}\right]\right)
\end{aligned}
$$

where $A_{1}$ and $A_{2}$ are unstable. Choosing $K_{1}$ and $K_{2}$ as

$$
K_{1}=\left[\begin{array}{cc}
5 & 0 \\
20 & 1
\end{array}\right], \quad K_{2}=\left[\begin{array}{cc}
-3 & -14 \\
2 & 4
\end{array}\right]
$$

then $A_{i}+B_{i} K_{i}$ for $i=1,2$ is Hurwitz. Although both subsystems are stable individually (for $\tau$ sufficiently small) as shown in Figure 6, switching between them may lead instability (Figure 8(a)). This is not unexpected; the same phenomenon has been observed in many switching systems involving ordinary differential equations $[9,25,26]$.

Set the Hurwitz linear convex combination $F$ by

$$
F=\frac{1}{3}\left(A_{1}+B_{1} K_{1}\right)+\frac{2}{3}\left(A_{2}+B_{2} K_{2}\right)=\left[\begin{array}{cc}
-2 & 22 / 3 \\
-28 / 3 & -2
\end{array}\right] .
$$

Hence, there exists a positive definite $P$

$$
P=\left[\begin{array}{cc}
46 / 489 & -3 / 1304 \\
-3 / 1304 & 293 / 3912
\end{array}\right], \quad \text { such that } F^{T} P+P F=-Q
$$




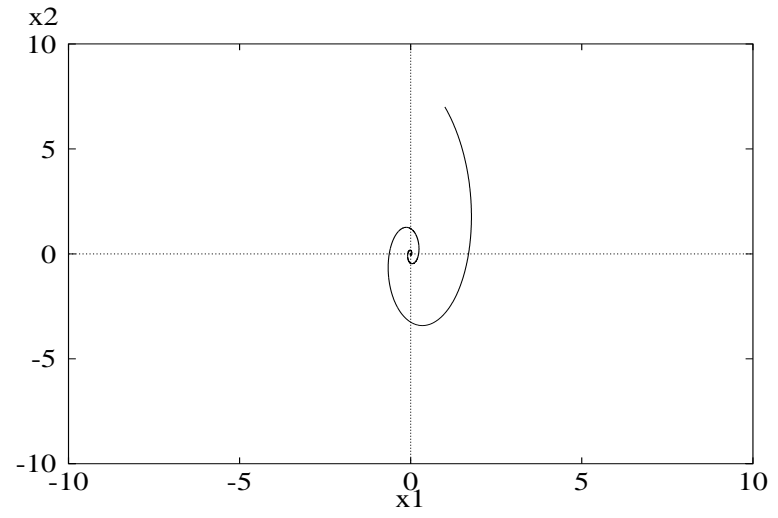

(a)

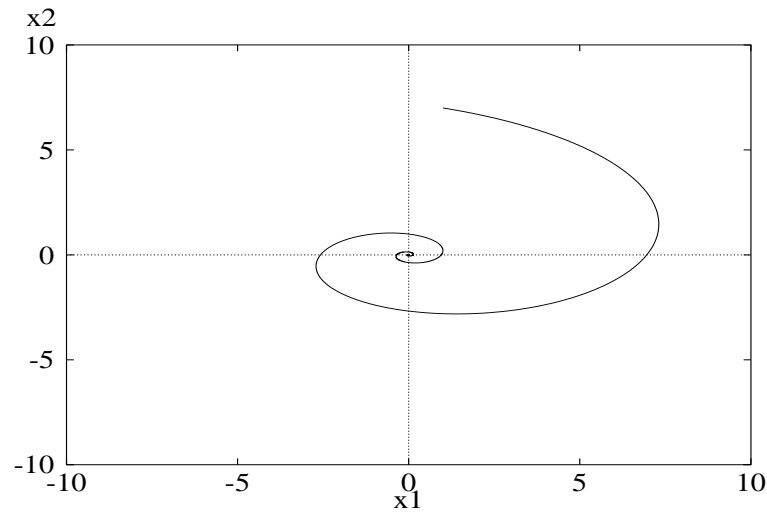

(b)

Figure 6: Behaviour of (a) mode 1 and (b) mode 2 of system (22) with $\tau=0.0087$. Initial condition: $\left(x_{1}(t), x_{2}(t)\right)=(1,7),-\tau \leq t \leq 0$.

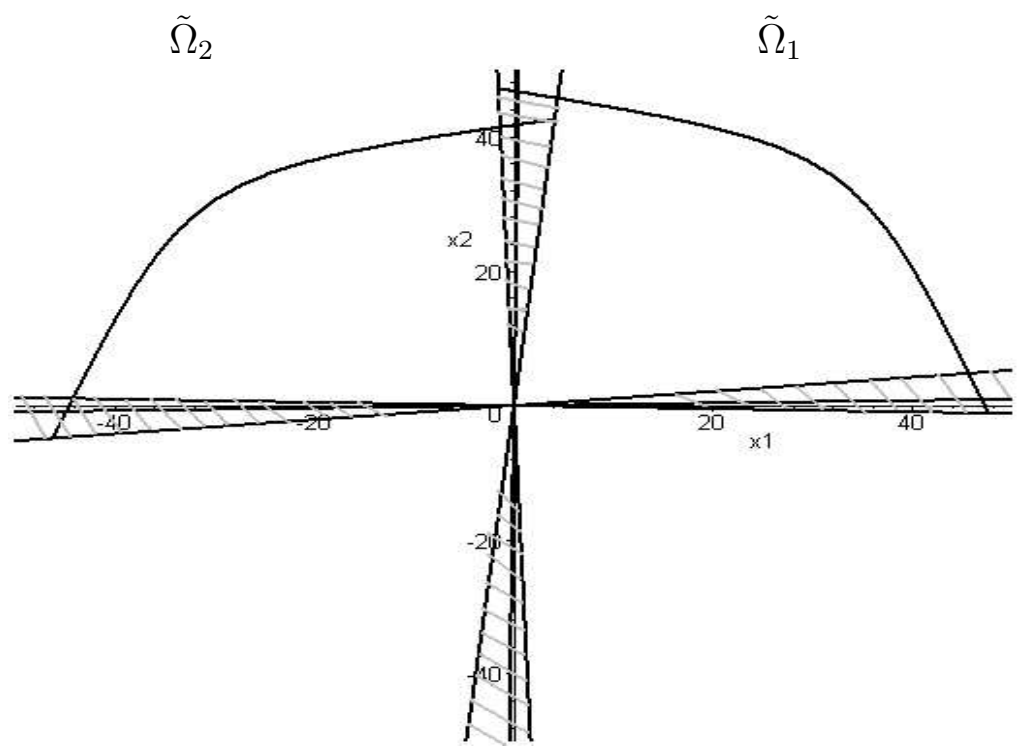

Figure 7: Switching regions for system (22): the shaded areas are overlapping regions 


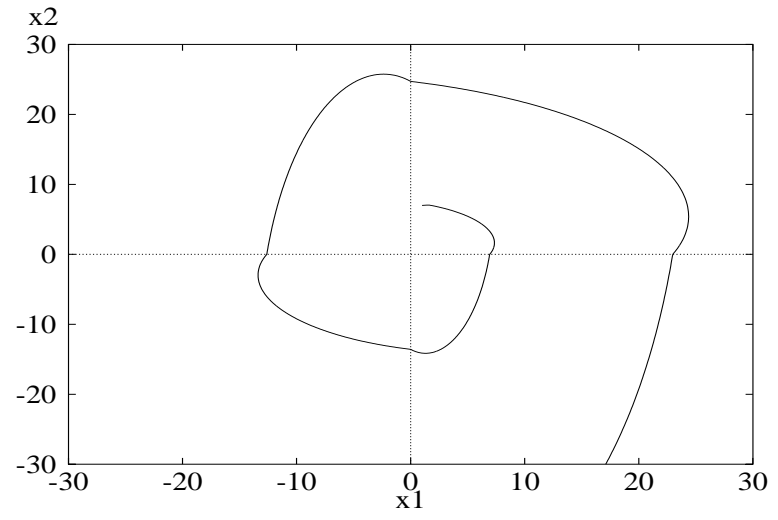

(a)

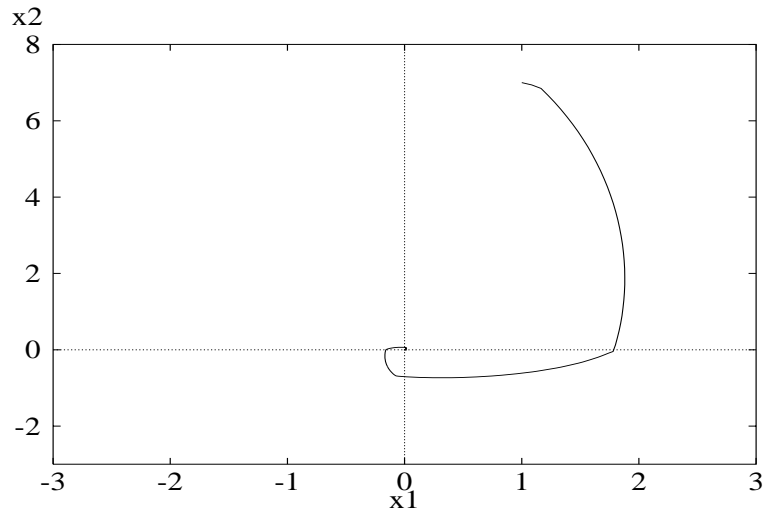

(b)

Figure 8: Comparison of switching rules applied to system (22) with $\tau=0.0087$. (a) Mode 1 in the 2 nd and 4th quadrant and mode 2 in the 1st and 3rd quadrant leads to instability. (b) Proposed switching rule gives asymptotic stability. Initial condition: $\left(x_{1}(t), x_{2}(t)\right)=$ $(1,7),-\tau \leq t \leq 0$.

where $Q=\frac{1}{3} I$. With this $P$ we construct switching regions for the mode 1 and mode 2 :

$$
\begin{aligned}
& \tilde{\Omega}_{1}=\left\{x \in \mathcal{R}^{2} \mid-(151 / 489) x_{2}^{2}-(424 / 163) x_{1} x_{2}-(139 / 489) x_{1}^{2} \leq-\frac{1}{4.5}\left(x_{1}^{2}+x_{2}^{2}\right)\right\}, \\
& \tilde{\Omega}_{2}=\left\{x \in \mathcal{R}^{2} \mid-(169 / 489) x_{2}^{2}+(212 / 163) x_{1} x_{2}-(175 / 489) x_{1}^{2} \leq-\frac{1}{4.5}\left(x_{1}^{2}+x_{2}^{2}\right)\right\},
\end{aligned}
$$

where $\xi=1.5$. These regions are illustrated in Figure 7. Choosing $R=S=19 I$, and $\xi=1.5$ in the Proposition 3 , we obtain the critical time delay, $\tau_{c} \approx 0.008723$. Then, Theorem 4 guarantees uniform asymptotic stability of (23) with the switching rule $\mathbf{S}$ for any $\tau<\tau_{c}$. This is illustrated in Figure 8(b). In fact, this delay estimate is conservative. In numerical simulations we observe stability up to $\tau=0.10591$, see Figure 9. 


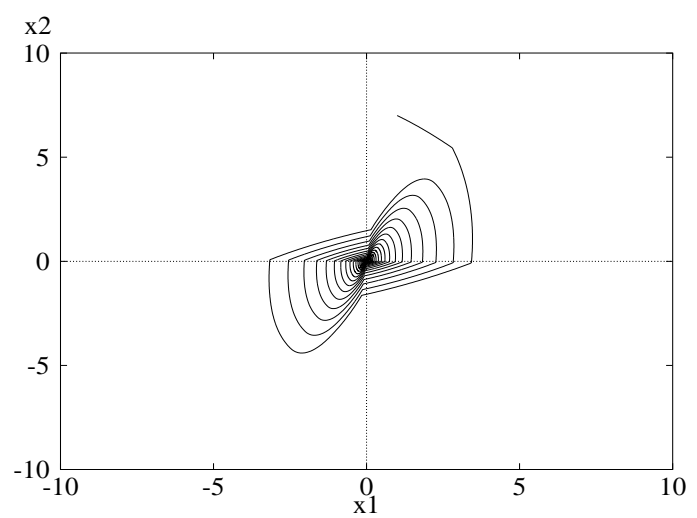

(a)

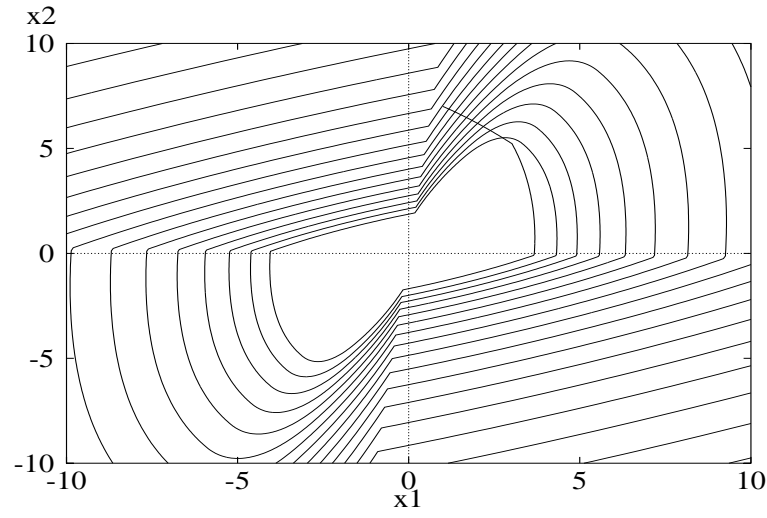

(b)

Figure 9: System (22) with proposed switching rule and various time delays: (a) $\tau=0.099$, (b) $\tau=0.11$. Initial condition: $\left(x_{1}(t), x_{2}(t)\right)=(1,7),-\tau \leq t \leq 0$.

\section{DISCUSSION}

In this paper we achieved uniform asymptotic stability of a class of linear switching systems with time delay by extending the result of ODE switching systems in [10] to a common Lyapunov functional method. The main advantages of our approach are as follows.

(i) We do not require that the coefficient matrix of the undelayed part of each subsystem $\left(A_{i}\right)$ to be Hurwitz.

(ii) We have quantified the size of delay needed for stability.

As has been observed with ODE systems [9, 25, 26], even if the delay is chosen such that each subsystem is stable, an ill-schemed switching rule can lead instability. This is illustrated in Figure 8(a) of Section III. Numerical simulations indicate that our condition on the delay is somewhat conservative: stability with our switching rule is maintained for delays larger than the theoretical value. Improving the theoretical result is an area for future work. Other possible avenues of future research include extending the model to include impulses at the switching moments.

\section{ACKNOWLEDGMENT}

The numerical simulations were done with XPPAUT [27] which is a software package that performs numerical integration of delay differential equations. 


\section{References}

[1] M. Branicky, V. Borkar, and S. Mitter, "A unified framework for hybrid control: model and optimal control theory," IEEE Trans. Automat. Contr., vol. 43, no. 1, pp. 31-45, 1998.

[2] D. Pepyne and C. Cassandaras, "Optimal control of hybrid systems in manufacturing," Proc. IEEE, vol. 88, no. 7, pp. 1008-1122, Jul. 2000.

[3] M. Song, T. Tran, and N. Xi, "Integration of task scheduling, action planning, and control in robotic manufacturing systems," Proc. IEEE, vol. 88, no. 7, pp. 1097-1107, Jul. 2000.

[4] R. Horowitz and P. Varaiya, "Control design of an automated highway system," Proc. IEEE, vol. 88, no. 7, pp. 913-925, Jul. 2000.

[5] C. Livadas, J. Lygeros, and N. A. Lynch, "High-level modeling and analysis of the traffic alert and collision avoidance system (TCAS)," Proc. IEEE, vol. 88, no. 7, pp. 926-948, Jul. 2000.

[6] P. Varaiya, "Smart cars on smart roads: Problems of control," IEEE Trans. Automat. Contr., vol. 38, no. 2, pp. 195-207, Feb. 1993.

[7] S. Engell, S. Kowalewski, C. Schulz, and O. Strusberg, "Continuous-discrete interactions in chemical processing plants," Proc. IEEE, vol. 88, no. 7, pp. 1050-1068, Jul. 2000.

[8] P. Antsaklis, "Special issue on hybrid systems: Theory and applications- a brief introduction to the theory and applications of hybrid systems," Proc. IEEE, vol. 88, no. 7, pp. 887-897, Jul. 2000.

[9] D. Liberzon, Switching in Systems and Control. Boston: Birkhäuser, 2003.

[10] M. Wicks, P. Peleties, and R. DeCarlo, "Switched controller synthesis for the quadratic stabilization of a pair of unstable linear systems," European Journal of Control, vol. 4, pp. 140-147, 1998.

[11] K. Gu, V. Kharitonov, and J. Chen, Stability of Time-delay Systems. Boston: Birkhäuser, 2003.

[12] V. Kolmanovskii and V. Nosov, Dynamics of Controlled Mechanical Systems with Delayed Feedback. New York: Springer-Verlag, 1986.

[13] Y. Kuang, Delayed Differential Equations with Applications in Population Dynamics. Boston: Academic Press, 1993.

[14] G. Stépán, Retarded Dynamical Systems, ser. Pitman Research Notes in Mathematics. Essex: Longman Group, 1989, vol. 210. 
[15] H. Wang, Dynamics of Controlled Mechanical Systems with Delayed Feedback. New York: Springer-Verlag, 2002.

[16] F. Gouaisbaut, W. Perruquetti, and J. Richard, "A sliding mode control for linear systems with input and state delays," in Proc. 38th IEEE Conference on Decision and Control, Phoenix, Arizona, USA, 1999, pp. 4234-4239.

[17] F. Gouaisbaut, M. Dambrine, and J. Richard, "Sliding mode control of TDS via functional surfaces," in Proc. 40th IEEE Conference on Decision and Control, Orlando, Florida, USA, 2001, pp. 4630-4634.

[18] X. Li and S. Yurkovich, Sliding mode Control of Systems with Delayed States and Controls, ser. Lecture note in control and information science. Berlin: Springer, 1999, vol. 247.

[19] X. Liu, X. Shen, and Y. Zhang, "Stability analysis of a class of hybrid dynamic systems," Dynamics of Continuous, Discrete and Impulsive Systems, Series B, vol. 8, no. 3, pp. 359-373, 2001.

[20] R. Yuan, S. Jing, and L. Chen, "Uniform asymptotic stability of hybrid dynamical systems with delay," IEEE Trans. Automat. Contr., vol. 48, no. 2, pp. 344-348, Feb. 2003.

[21] E.-K. Boukas and Z.-K. Liu, Deterministic and Stochastic Time Delay Systems. Boston: Birkhäuser, 2002.

[22] J. Hale and S. V. Lunel, Introduction to Functional Differential Equations. New York: Springer-Verlag, 1993.

[23] V. Kolmanovskii and J. Richard, "Stability of some linear systems with delays," IEEE Trans. Automat. Contr., vol. 44, no. 5, pp. 984-989, May 1998.

[24] S. Kim, "Stability of switching systems with delay," Ph.D. dissertation, University of Waterloo, 2005.

[25] M. Branicky, "Multiple Lyapunov functions and other analysis tools for switched and hybrid systems," IEEE Transactions on Automatic Control, vol. 43, no. 4, pp. 475-482, April 1998.

[26] M. Johansson and A. Rantzer, "Computation of piecewise quadratic Lyapunov functions for hybrid systems," IEEE Transactions on Automatic Control, vol. 43, no. 4, pp. 555-559, April 1998.

[27] B. Ermentrout, Simulating, Analyzing and Animating Dynamical Systems: A Guide to XPPAUT for Researcher and Students. Philadelphia: SIAM, 2002. 


\section{APPENDIX: Proof of Proposition 3}

Assume that $x(t)$ is in $\tilde{\Omega}_{i}$ on $\left[t_{k}, t_{k+1}\right)$ for some $k \in\{0,1,2, \cdots\}$ and switched from the $j^{\text {th }}$ mode at $t_{k}$. From (13) we have

$$
\begin{gathered}
\dot{V}\left(x_{t}\right)=\dot{x}(t)^{T} P x(t)+x^{T}(t) P \dot{x}(t)+\int_{\tau}^{2 \tau}\left[x^{T}(t) R x(t)-x^{T}(t-s) R x(t-s)\right] d s \\
+\int_{0}^{\tau}\left[x^{T}(t) S x(t)-x^{T}(t-s) S x(t-s)\right] d s .
\end{gathered}
$$

As discussed on pp. 6-7, the expression for $\dot{x}(t)$ depends on whether $\tau$ is greater than or less than $t_{k+1}-t_{k}$. We thus divide the rest of the proof into two cases.

Case 1. $t_{k+1}-t_{k} \leq \tau$

In this case the mixed mode covers to the whole switching interval $\left[t_{k}, t_{k+1}\right)$, i.e. $\dot{x}(t)$ is given by (18). Thus, for $t \in\left[t_{k}, t_{k+1}\right)$, we have

$$
\begin{aligned}
\dot{V}\left(x_{t}\right)= & \left(\left(A_{i}+B_{i}\right) x(t)-B_{i} \int_{t-\tau}^{t_{k-m+1}}\left(A_{p_{m}} x(s)+B_{p_{m}} x(s-\tau)\right) d s\right. \\
& \left.\quad-B_{i} \sum_{j=1}^{m-1} \int_{t_{k-j}}^{t_{k-j+1}}\left(A_{p_{j}} x(s)+B_{p_{j}} x(s-\tau)\right) d s-B_{i} \int_{t_{k}}^{t}\left(A_{i} x(s)+B_{i} x(s-\tau)\right) d s\right)^{T} P x(t) \\
+ & x^{T}(t) P\left(\left(A_{i}+B_{i}\right) x(t)-B_{i} \int_{t-\tau}^{t_{k-m+1}}\left(A_{p_{m}} x(s)+B_{p_{m}} x(s-\tau)\right) d s\right. \\
& \left.\quad-B_{i} \sum_{j=1}^{m-1} \int_{t_{k-j}}^{t_{k-j+1}}\left(A_{p_{j}} x(s)+B_{p_{j}} x(s-\tau)\right) d s-B_{i} \int_{t_{k}}^{t}\left(A_{i} x(s)+B_{i} x(s-\tau)\right) d s\right) \\
+ & \int_{\tau}^{2 \tau}\left(x^{T}(t) R x(t)-x^{T}(t-s) R x(t-s)\right) d s+\int_{0}^{\tau}\left(x^{T}(t) S x(t)-x^{T}(t-s) S x(t-s)\right) d s .
\end{aligned}
$$

It follows that

$$
\begin{gathered}
\dot{V}\left(x_{t}\right)=x^{T}(t)\left(\left(A_{i}+B_{i}\right)^{T} P+P\left(A_{i}+B_{i}\right)\right) x(t)+\tau x^{T}(t)(R+S) x(t) \\
-\int_{t-\tau}^{t_{k-m+1}}\left(x^{T}(s)\left(B_{i} A_{p_{m}}\right)^{T} P x(t)+x^{T}(s-\tau)\left(B_{i} B_{p_{m}}\right)^{T} P x(t)\right) d s \\
\quad-\int_{t-\tau}^{t_{k-m+1}}\left(x^{T}(t) P B_{i} A_{p_{m}} x(s)+x^{T}(t) P B_{i} B_{p_{m}} x(s-\tau)\right) d s \\
-\sum_{j=1}^{m-1} \int_{t_{k-j}}^{t_{k-j+1}}\left(x^{T}(s)\left(B_{i} A_{p_{j}}\right)^{T} P x(t)+x^{T}(s-\tau)\left(B_{i} B_{p_{j}}\right)^{T} P x(t)\right) d s \\
\quad-\sum_{j=1}^{m-1} \int_{t_{k-j}}^{t_{k-j+1}}\left(x^{T}(t) P B_{i} A_{p_{j}} x(s)+x^{T}(t) P B_{i} B_{p_{j}} x(s-\tau)\right) d s \\
-\int_{t_{k}}^{t}\left(x^{T}(s)\left(B_{i} A_{i}\right)^{T} P x(t)+x^{T}(s-\tau)\left(B_{i}^{2}\right)^{T} P x(t)\right) d s \\
\quad-\int_{t_{k}}^{t}\left(x^{T}(t) P B_{i} A_{i} x(s)+x^{T}(t) P B_{i}^{2} x(s-\tau)\right) d s \\
-\int_{t-\tau}^{t_{k-m+1}} x^{T}(s) S x(s) d s-\sum_{j=1}^{m-1} \int_{t_{k-j}}^{t_{k-j+1}} x^{T}(s) S x(s) d s-\int_{t_{k}}^{t} x^{T}(s) S x(s) d s \\
-\int_{t-\tau}^{t_{k-m+1}} x^{T}(s-\tau) R x(s-\tau) d s-\sum_{j=1}^{m-1} \int_{t_{k-j}}^{t_{k-j+1}} x^{T}(s-\tau) R x(s-\tau) d s \\
\quad-\int_{t_{k}}^{t} x^{T}(s-\tau) R x(s-\tau) d s .
\end{gathered}
$$

Using the fact that $x^{T}(t)\left(\left(A_{i}+B_{i}\right)^{T} P+P\left(A_{i}+B_{i}\right)\right) x(t) \leq-\frac{1}{\xi} x^{T}(t) Q x(t)$ and adding and 
subtracting the following integrals

$$
\begin{array}{ll}
\int_{t-\tau}^{t_{k-m+1}} x^{T}(t) P B_{i} A_{p_{m}} S^{-1}\left(B_{i} A_{p_{m}}\right)^{T} P x(t) d s, & \int_{t-\tau}^{t_{k-m+1}} x^{T}(t) P B_{i} B_{p_{m}} R^{-1}\left(B_{i} B_{p_{m}}\right)^{T} P x(t) d s, \\
\sum_{j=1}^{m-1} \int_{t_{k-j}}^{t_{k-j+1}} x^{T}(t) P B_{i} A_{p_{j}} S^{-1}\left(B_{i} A_{p_{j}}\right)^{T} P x(t) d s, & \sum_{j=1}^{m-1} \int_{t_{k-j}}^{t_{k-j+1}} x^{T}(t) P B_{i} B_{p_{j}} R^{-1}\left(B_{i} B_{p_{j}}\right)^{T} P x(t) d s, \\
\int_{t_{k}}^{t} x^{T}(t) P B_{i} A_{i} S^{-1}\left(B_{i} A_{i}\right)^{T} P x(t) d s, & \int_{t_{k}}^{t} x^{T}(t) P B_{i}^{2} R^{-1}\left(B_{i}^{2}\right)^{T} P x(t) d s,
\end{array}
$$

we obtain

$$
\begin{aligned}
\dot{V}\left(x_{t}\right) \leq & -\frac{1}{\xi} x^{T}(t) Q x(t)+\tau x^{T}(t)(R+S) x(t) \\
& -\int_{t-\tau}^{t_{k-m+1}}\left[\left(P B_{i} A_{p_{m}}\right)^{T} x(t)+S x(s)\right]^{T} S^{-1}\left[\left(P B_{i} A_{p_{m}}\right)^{T} x(t)+S x(s)\right] d s \\
& -\int_{t-\tau}^{t_{k-m+1}}\left[\left(P B_{i} B_{p_{m}}\right)^{T} x(t)+R x(s-\tau)\right]^{T} R^{-1}\left[\left(P B_{i} B_{p_{m}}\right)^{T} x(t)+R x(s-\tau)\right] d s \\
& -\sum_{j=1}^{m-1} \int_{t_{k-j}}^{t_{k-j+1}}\left[\left(P B_{i} A_{p_{j}}\right)^{T} x(t)+S x(s)\right]^{T} S^{-1}\left[\left(P B_{i} A_{p_{j}}\right)^{T} x(t)+S x(s)\right] d s \\
& -\sum_{j=1}^{m-1} \int_{t_{k-j}}^{t_{k-j+1}}\left[\left(P B_{i} B_{p_{j}}\right)^{T} x(t)+R x(s-\tau)\right]^{T} R^{-1}\left[\left(P B_{i} B_{p_{j}}\right)^{T} x(t)+R x(s-\tau)\right] d s \\
& -\int_{t_{k}}^{t}\left[\left(P B_{i} A_{i}\right)^{T} x(t)+S x(s)\right]^{T} S^{-1}\left[\left(P B_{i} A_{i}\right)^{T} x(t)+S x(s)\right] d s \\
& -\int_{t_{k}}^{t}\left[\left(P B_{i}^{2}\right)^{T} x(t)+R x(s-\tau)\right]^{T} R^{-1}\left[\left(P B_{i}^{2}\right)^{T} x(t)+R x(s-\tau)\right] d s \\
+ & \int_{t-\tau}^{t_{k-m+1}} x^{T}(t) P B_{i} A_{p_{m}} S^{-1}\left(B_{i} A_{p_{m}}\right)^{T} P x(t) d s+\int_{t-\tau}^{t_{k-m+1}} x^{T}(t) P B_{i} B_{p_{m}} R^{-1}\left(B_{i} B_{p_{m}}\right)^{T} P x(t) d s \\
+ & \sum_{j=1}^{m-1} \int_{t_{k-j}}^{t_{k-j+1}} x^{T}(t) P B_{i} A_{p_{j}} S^{-1}\left(B_{i} A_{p_{j}}\right)^{T} P x(t) d s \\
& \quad+\sum_{j=1}^{m-1} \int_{t_{k-j}}^{t_{k-j}} x^{T}(t) P B_{i} B_{p_{j}} R^{-1}\left(B_{i} B_{p_{j}}\right)^{T} P x(t) d s \\
+ & \int_{t_{k}}^{t} x^{T}(t) P B_{i} A_{i} S^{-1}\left(B_{i} A_{i}\right)^{T} P x(t) d s+\int_{t_{k}}^{t} x^{T}(t) P B_{i}^{2} R^{-1}\left(B_{i}^{2}\right)^{T} P x(t) d s .
\end{aligned}
$$

Dropping the negative integrals then gives

$$
\begin{aligned}
\dot{V}\left(x_{t}\right) \leq & -\frac{1}{\xi} \lambda_{\min }(Q)\|x(t)\|^{2}+\tau\left(\lambda_{\max }(R)+\lambda_{\max }(S)\right)\|x(t)\|^{2} \\
& +\left(t_{k-m+1}-(t-\tau)\right) x^{T}(t)\left(P B_{i}\left(A_{p_{m}} S^{-1} A_{p_{m}}^{T}+B_{p_{m}} R^{-1} B_{p_{m}}^{T}\right) B_{i}^{T} P\right) x(t) \\
& +\sum_{j=1}^{m-1}\left(t_{k-j+1}-t_{k-j}\right) x^{T}(t)\left(P B_{i}\left(A_{p_{j}} S^{-1} A_{p_{j}}^{T}+B_{p_{j}} R^{-1} B_{p_{j}}^{T}\right) B_{i}^{T} P\right) x(t) \\
& +\left(t-t_{k}\right) x^{T}(t)\left(P B_{i}\left(A_{i} S^{-1} A_{i}^{T}+B_{i} R^{-1} B_{i}^{T}\right) B_{i}^{T} P\right) x(t) .
\end{aligned}
$$


And hence, we have

$$
\begin{aligned}
\dot{V}\left(x_{t}\right) \leq & -\frac{1}{\xi} \lambda_{\min }(Q)\|x(t)\|^{2}+\tau\left(\lambda_{\max }(R)+\lambda_{\max }(S)\right)\|x(t)\|^{2} \\
& +\left(t_{k-m+1}-(t-\tau)\right) \lambda_{\max }\left(P B_{i}\left(A_{p_{m}} S^{-1} A_{p_{m}}^{T}+B_{p_{m}} R^{-1} B_{p_{m}}^{T}\right) B_{i}^{T} P\right)\|x(t)\|^{2} \\
& +\left(\sum_{j=1}^{m-1}\left(t_{k-j+1}-t_{k-j}\right) \lambda_{\max }\left(P B_{i}\left(A_{p_{j}} S^{-1} A_{p_{j}}^{T}+B_{p_{j}} R^{-1} B_{p_{j}}^{T}\right) B_{i}^{T} P\right)\|x(t)\|^{2}\right. \\
& +\left(t-t_{k}\right) \lambda_{\max }\left(P\left(A_{i} S^{-1} A_{i}^{T}+B_{i} R^{-1} B_{i}^{T}\right) B_{i}^{T} P\right)\|x(t)\|^{2} \\
\leq & -\frac{1}{\xi} \lambda_{\min }(Q)\|x(t)\|^{2}+\tau\left(\lambda_{\max }(R)+\lambda_{\max }(S)\right)\|x(t)\|^{2} \\
& +\left(t_{k-m+1}-(t-\tau)\right) \lambda_{\max }^{i}\|x(t)\|^{2}+\sum_{j=1}^{m-1}\left(t_{k-j+1}-t_{k-j}\right) \lambda_{\max }^{i}\|x(t)\|^{2}+\left(t-t_{k}\right) \lambda_{\text {max }}^{i}\|x(t)\|^{2} \\
= & -\frac{1}{\xi} \lambda_{\min }(Q)\|x(t)\|^{2}+\tau\left(\lambda_{\max }(R)+\lambda_{\max }(S)\right)\|x(t)\|^{2} \\
& +\left(\left(t_{k-m+1}-(t-\tau)\right)+\sum_{j=1}^{m-1}\left(t_{k-j+1}-t_{k-j}\right)+\left(t-t_{k}\right)\right) \lambda_{\max }^{i}\|x(t)\|^{2} \\
= & -\left(\frac{1}{\xi} \lambda_{\min }(Q)-\tau\left(\lambda_{\max }(R)+\lambda_{\max }(S)+\lambda_{\max }^{i}\right)\right)\|x(t)\|^{2} \\
\leq & -\gamma\left(\left\|x_{t}(0)\right\|\right),
\end{aligned}
$$

where $\gamma(s)=\left(\frac{1}{\xi} \lambda_{\min }(Q)-\tau L\right) s^{2}$. The properties of $\gamma$ are clear by the choice of the delay.

Case 2. $t_{k+1}-t_{k}>\tau$

In this case we need to consider two subintervals: $\left[t_{k}, t_{k}+\tau\right)$ and $\left[t_{k}+\tau, t_{k+1}\right)$. For $t \in\left[t_{k}, t_{k}+\tau\right), \dot{x}(t)$ is given by (18) and the analysis is same as for Case 1. For $t \in\left[t_{k}+\tau, t_{k+1}\right), \dot{x}(t)$ is given by (17) and we have

$$
\begin{aligned}
\dot{V}\left(x_{t}\right)=x^{T}( & (t)\left(\left(A_{i}+B_{i}\right)^{T} P+P\left(A_{i}+B_{i}\right)\right) x(t)+\tau x^{T}(t)(R+S) x(t) \\
- & 2 \int_{t-\tau}^{t} x^{T}(t) P B_{i} A_{i} x(s) d s-\int_{t-\tau}^{t} x^{T}(s) S x(s) d s \\
& \quad-2 \int_{t-\tau}^{t} x^{T}(t) P B_{i}^{2} x(s-\tau) d s-\int_{t-\tau}^{t} x^{T}(s-\tau) R x(s-\tau) d s .
\end{aligned}
$$

Following a similar procedure as for Case 1, we find

$$
\begin{aligned}
& \dot{V}\left(x_{t}\right) \leq-\frac{1}{\xi} x^{T}(t) Q x(t) \\
&+\tau x^{T}(t)\left\{R+S+P B_{i}\left(A_{i} S^{-1} A_{i}^{T}+B_{i} R^{-1} B_{i}^{T}\right) B_{i}^{T} P\right\} x(t) \\
& \quad-\int_{t-\tau}^{t}\left[\left(B_{i} A_{i}\right)^{T} P x(t)+S x(s)\right]^{T} S^{-1}\left[\left(B_{i} A_{i}\right)^{T} P x(t)+S x(s)\right] d s \\
& \quad \quad-\int_{t-\tau}^{t}\left[\left(B_{i}^{2}\right)^{T} P x(t)+R x(s-\tau)\right]^{T} R^{-1}\left[\left(B_{i}^{2}\right)^{T} P x(t)+R x(s-\tau)\right] d s \\
& \leq \quad-\frac{1}{\xi} \lambda_{\min }(Q)\|x(t)\|^{2}+\tau\left\{\lambda_{\max }(R)+\lambda_{\max }(S)\right. \quad+\lambda_{\max }\left(P B_{i}\left(A_{i} S^{-1} A_{i}^{T}+B_{i} R^{-1} B_{i}^{T} P\right) B_{i}^{T} P\right\}\|x(t)\|^{2} \\
& \leq \quad-\frac{1}{\xi} \lambda_{\min }(Q)\|x(t)\|^{2}+\tau\left(\lambda_{\max }(R)+\lambda_{\max }(S)+\lambda_{\max }^{i}\right)\|x(t)\|^{2} \\
& \leq-\gamma\left(\left\|x_{t}(0)\right\|\right) .
\end{aligned}
$$

This completes the proof. 